\title{
An Interpretation of Quantum Entanglement and the Nature of the Universe
}

\author{
Jun Steed HUANG ${ }^{1,2, a,{ }^{,},}$Jiamin Moran HUANG ${ }^{1, b}$ and Guang-Qian LU ${ }^{2, c}$ \\ ${ }^{1} 399$ South Huanghe, Suqian, Jiangsu 223800, China \\ ${ }^{2} 161$ Louis Pasteur, Ottawa, Ontario K1N 6N5, Canada \\ a steedhuang@ujs.edu.cn, ’bmoranhgirl@gmail.com, 9guan077@uottawa.ca \\ ${ }^{*}$ Corresponding author
}

Keywords: Quantum, Space, Time, Encryption.

\begin{abstract}
Albert Einstein found that, for two particles coming from the same source, when the state of one changes, that of the other may change at the same time, no matter how far they are apart from each other. This superluminal phenomenon totally violates the special theory of relativity which says nothing's speed exceeds the limit of light speed, what's more, as of today, nobody has made a satisfying explanation of this strange phenomenon, not even Einstein's own general relativity. We bring forward our novel view of this long pending dilemma based on the observations of the latest physical lab reports and by summarizing various theories of information, computer science, communications, religion and physics itself. We call it Super General Relativity for now.
\end{abstract}

\section{Introduction}

In quantum physics, after two particles coupling for a short time, disturbing one of them would definitely affects the state of another one, even if they are far away from each other. This phenomenon is called the quantum entanglement. And its effects have been demonstrated with photons, electrons, molecules, and even small diamonds. Quantum entanglement occurs in the quantum system containing more than two particles. Quantum entanglement is a phenomenon which only happens in quantum system and it contradicts classical physics. We introduce following five axioms to remove the contradiction and explain this wired phenomenon:

1. $U \leftarrow M \& E$; World is made up of material and its associated energy (based on dialectical materialism).

2. $\mathrm{M}+\mathrm{E} \rightarrow \mathrm{S}$; Material movement with its energy makes us the space (this is the core of our conjecture).

3. $\Delta \mathrm{S} \rightarrow \mathrm{I}$; Material have different state in the space made by themselves (the state is information and consciousness alike).

4. $\Delta \mathrm{I} \rightarrow \mathrm{T}$; The relatively change of the state brings us time (this core idea explain why the slowly changing physiology state of turtle gets them long lifespan).

5 . $\mathrm{M}^{*} \mathrm{~S} 1 * \mathrm{~S} 2=\mathrm{E}^{*} \mathrm{~T} 1 * \mathrm{~T} 2$; The quantity of material determines the space size, the time pace and the energy capacity (material space and energy time super conservation).

Einstein's special theory of relativity and general relativity united the indivisible internal connection between space and time or material and energy. But he didn't point out that there is much more indivisible connection between space and material as well: the essence of the world, the meaning of philosophy's existence, the energy field said by the master of ancient meditation or the fortune-teller of metaphysics, and the reason of the what you did would always come back to you emphasized by the five religions (Buddhism, Taoism, Islam, Papistry and Christianity). 


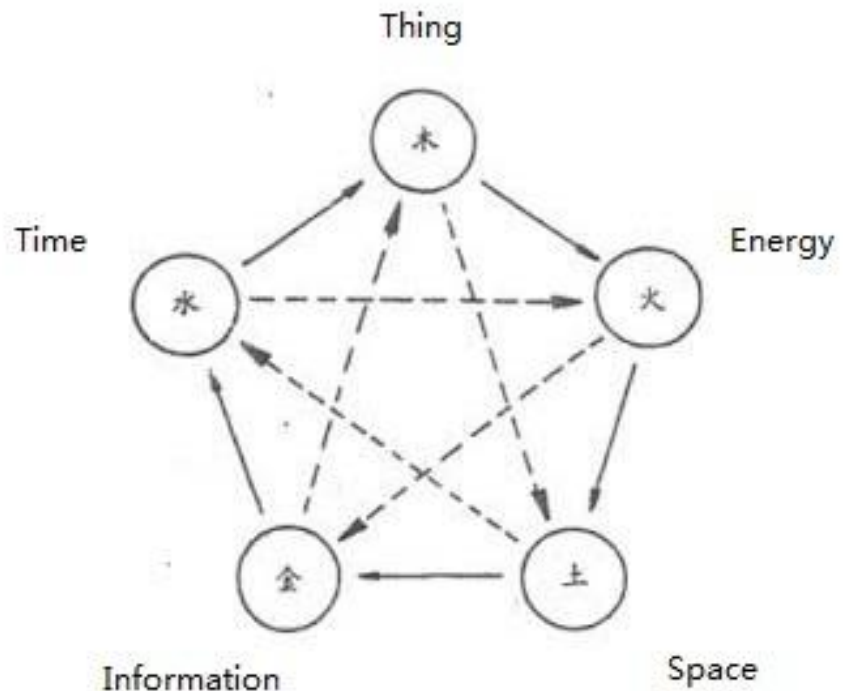

Figure 1. Ancient Five Elements Culture and Relativity Science

The five elements in Fig. 1 can be described as following: wood is material or thing, wood makes fire, fire is energy, fire creates dust, dust is space, dust creates gold, gold is state or information, gold creates water, water is time, water creates wood, and the loop goes on. Our super general relativity is about the infinite circle between material, energy, space, information and time. Solid line is positive forward relationship, dashed line is negative backward relationship, emphasized by Chinese culture.

First we interpreted the quantum entanglement phenomenon with our thing, energy, space, information, time relationship assumption, then we will prove and discuss it based on existed wellknown physical lab reports with more interesting thought experiments.

\section{Using Super General Relativity to Interpret Quantum Entanglement}

After particles contacting to each other for a short time, they may be coupling successfully and quantum entanglement occurs, or failed. Whether quantum entanglement occurs or not is decided by many factors, but the key factor is whether the space is relatively thin or not. If it is thin, then the answer is certainly yes. Many physical experiments [1] have proven that the probability of quantum entanglement occurring in space lab in thin space is high than that in ground lab in thick space. That's why we use quantum encryption on telecommunications satellites more than on ground quantum computers. In another aspect, we can calculate the probability of the occurring of quantum entanglement to judge the space density itself.

For the particles which are involved with quantum entanglement, when something disturbs/changes one of them, another one would certainly be affected, because they are in the space made together by them with the natural state they are. And when we disturbs/changes one of them, we disturbs/changes the space made by both of them, that equals to disturbing/changing the space made by another particle. Although the particles may be separated by a long distance between each other, the changing of their state synchronizes, because of the trinity of their space and themselves, just like when grab one head of magic stick owned by the power monkey (Fig. 2) and twist it with 90 degrees, the other end of it would spin 90 degrees immediately too. 


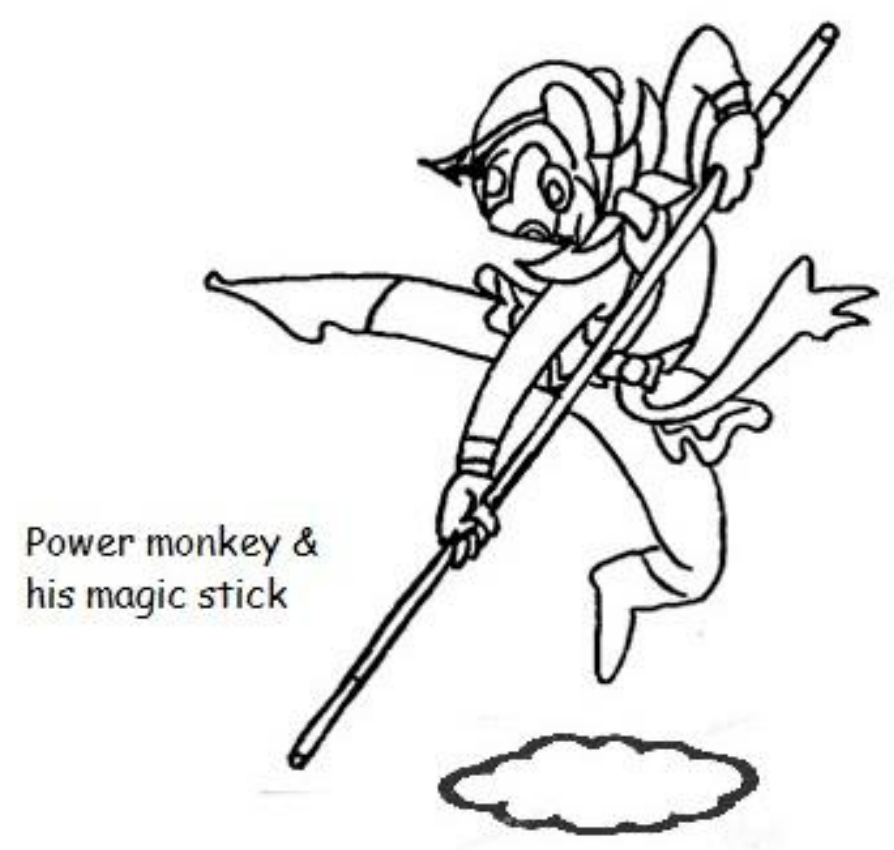

Figure 2. Size Changeable Magic Stick of Flying Monkey King

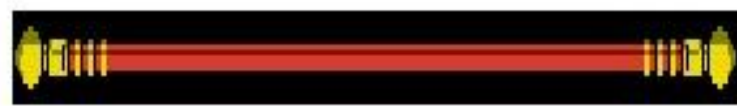

\section{Magic stick made of their own space}

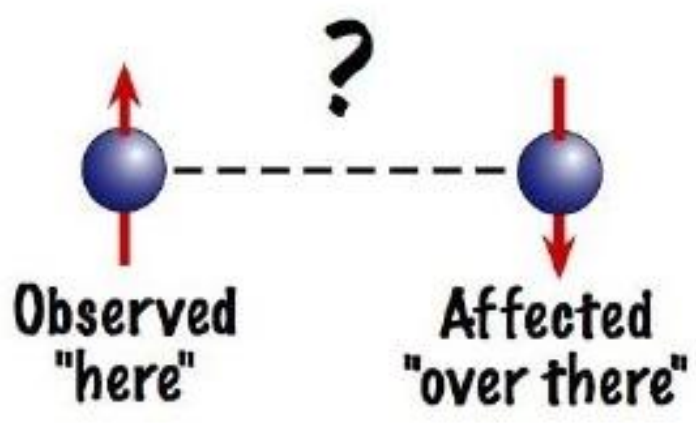

Figure 3. Quantum Entanglement Rooted from the Magic Space Created

Attention, the magic stick could turn into a really tiny size which could even fit into his ear or become big enough to support the south sky. And this is the size changing on space we are talking about, when two particles are pulled apart [Fig.3], but remains entangled. The phenomenon is what we understand about quantum entanglement ---- the process of magic space creating. Quantum entanglement could occur between the macroscopic particles or the microscopic particles because they are both important in creating space. We will tell more thought experiments in following to help understanding of the five simple axioms made in the Introduction part.

\section{How to Produce More Entangled Photons ---- Challenges in the Quantum Encryption}

The article on ERP paradox written by Albert Einstein, Boris Podolsky and Nathan Rosen in 1935 involves problems caused by two particles' quantum entanglement from where the research on quantum entanglement starts. In the same year, Erwin Schroedinger published some papers about 
quantum entanglement too, and he named this phenomenon quantum entanglement. Although these researchers kept eyes on revealing quantum physics by claiming the behavior violated the local realist view of causality. However, the experiments on this subject all prove the theoretical predicts are right and make most physicists admit that quantum entanglement is the basis of quantum physics. Now, the core of this research is turned into application stage, such as secure communication, quantum computer science and so on. However, physicists still know little about the underlying mechanism of the quantum entanglement.

And we give you the tentative thought experiment and our philosophical answer about it: what behind the quantum entanglement is space itself! So, if you want more entangled photons you have to be at a place with less persons or make the space thin, such as the cold atom cavity mentioned by reference [2] and so on. Attention, the space we are talking about is not the Ether defined many years ago, because the ether theory assumes space a special, independent even material which can't be modified. On contrary, our opinion is that space is not material, not even and absolutely not independent. Space and material is like you and your shadow, they never leave each other and the space we live in is a big space that made up with small spaces. And this philosophy can explain why masters of fortune teller care about the setting of furniture so much ---- every change of things in your room would change the feature of the space that you live in.

\section{Superluminal Phenomenon ---- Space's Extension rather than Flying Photon}

Assume a complex system with two particles interacting in quantum state, for the data we get by testing one of the particles (assume it's right spin), we can get the relative data by testing another particle's state in any time (at here is left spin). By testing each pair of particles in a series of entangled particles, we can tell a relative phenomenon between each pair of particles by the data. Even if they are long distance away from each other, we can still see the phenomenon. We consider the basis of the relationship as the flipping of space itself. Experiences have been done to verify the relationship. The phenomenon happens even when the time interval taken is shorter than the time light flies between two particles. In other words, quantum entanglement is faster than light. And the experience recently completed in reference [3] presents that the speed of quantum entanglement is at least 10,000 times faster than light and it's only the lower limit. Then, what is its upper limit?

A monk from India made a speech at University of California, USA. He pointed out that what sun broadcasts is space but not light. We admire the philosophical view and would like to expand it. According to our super general relativity thinking, the stiffness of space decides the speed of quantum entanglement. The stiffness is inversely proportional to the density of space. The thinner the space is, the stronger the stiffness is; the less time to flip the space (think it as magic stick used by powerful monkey) takes, the faster the action is. The two experiments as following prove the logic: experiment one was done on a mountain in Geneva (Europe), experiment two was done on sea level in Qingdao (Asia), and the gravity field (space density) of Qingdao is certainly stronger than that of Geneva.

Experiment one: In 2013, Chinese scientists experimentally found that, quantum entanglement is 10,000 times faster than the speed of light near Qingdao. And They further found the speed is the lower limit of action after more experiments;

Experiment two: In 2008, researchers found that the quantum entanglement in their experimental installation is much faster than 10,000 times of speed of light, reference [4].

According to our super general relativity, only when stiffness of space is close to zero (like on high orbit geostationary satellites), in other word, in the situation of nearly no space, the measurable effect would be close to the absolutely momentarily up limit. 


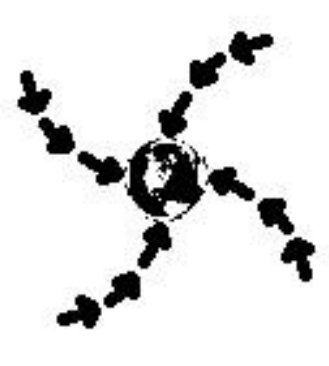

Lobachevsky -

Riemann space

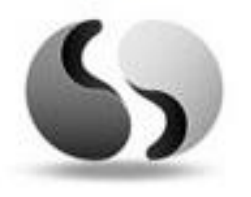

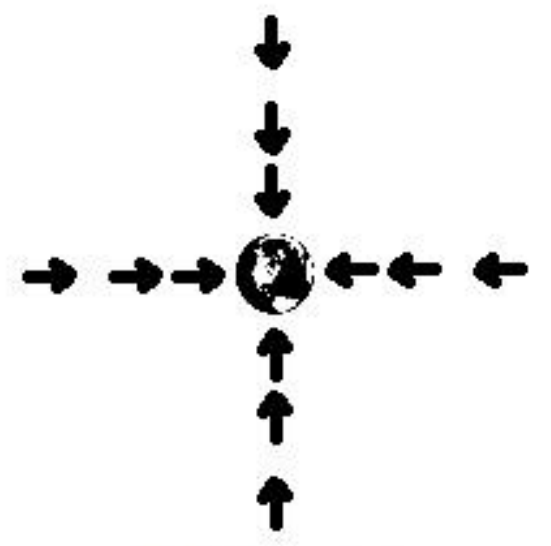

Euclidean space

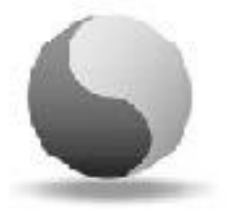

Figure 4. Spin Universe View rather than Big Bang Illusion

Experiments also prove that the broadcast distance of quantum entanglement traveling in space satellite communication is far more than the same system on the ground.

\section{Half of the Big Bang Theory is Right?}

The big bang theory is based on Einstein's general relativity and it simplified the solution of the field equations a little bit (as the independence, equality and isotropic of space). The big bang theory cosmologist thinks that the universe expanded from an extremely dense and hot state (the universe predicts a calculated age of $137.98 \pm 0.37$ billion years according to the observational result of Planck satellite in 2013) and continues to expand.

Space is created by material instead of being an independent system in our view, and because of the non-uniform of material distribution, space would never be uniform and isotropic, and, thus meet the precursor of the big-bang solution of the equations. The Milky galaxy is like a dish shape -- only a two-dimensional space observed from far away, so our space density can not be the same in any direction.

In 1922, USSR physicist Alexander Friedman described the fluid with the general relativity and proposed the field equations. In 1927, Rome solved the equations and brought forward the same view, and this solution called Friedmann-Lemaitre-Robertson-Walker metric. In 1929, American physicist Edwin Hubble discovered that all the galaxy and nebula are leaving away from the view point of us in sight velocity and the further they go the faster they are (Fig. 4 right side) in Euclidean space. If the distance of galaxy and nebula now is getting bigger, they could be really close to each other in the past. Physicists confer further from this illusion that the universe was in an extremely dense and hot state.

However, all the deep space detection vehicles didn't get any direct evidence back on expansion of the universe during past decades traveling. We give the explanation that the big bang never happened and the galaxy spectrum's redshift (wavelength gets longer) is only the heterogeneous density nature of universe. Because in our theory, the density and orientation of space is decided by material movement, when the mass of galaxy is in circular motion, the space around getting behind each other (Fig.4 left part) and makes it looks like it is expanding, but not really in Lobachevsky or Riemann space.

Although the results of experiments did in big particle accelerators support the big bang theory 
indirectly, the particle accelerators we get now is really limited on the range of high energy, so we have no evidence to describe the universe in the initial state before it gets extended. And because of this, the big bang theory still can't make any description or explanation on the universe's first stage. Actually, what it can describe and guess is the evolution picture after the big bang, if there was one.

Big Bang is a word proposed by English astronomer Fred Hoyle. He is actually the advocator of the oppose theory----steady state theory. The cosmic microwave background radiation found in 1964 is the evidence to support the big bang theory, especially after measuring the spectrum of it and making the curve of its black-body prediction, part of the scientists started to follow the big bang theory.

But in our opinion, the background radiation is only the result of partial explosion instead of the entire universe's explosion, because of the picture of cosmic microwave background radiation's unevenness. In another word, the universe will never meet together at a point, it takes time for space to expand and meet each other. The big bang theory can't stand on the ground of philosophy too; a problem brought by it is what's universe like before the big bang? The core of the big bang theory is that the explosion brings the space and the time, and we think the big bang never happened, but tiny or micro kind of explosion is happening at all times and each pair of particles which starts entangling [5] is creating magic micro space and micro time.

\section{Summary}

The assumption of axioms and thought experiments above declares a material-space based view from us:

1. Superluminal is entangled state's magic shadowing space;

2. Quantum encryption should be done in place with less gravitation or vacuum;

3. Time and space is made from some kind of explorations but not one big bang.

In general, we think that the universe have moving boundaries, the basis of space is in spin; the big bang never happens, micro explosion never stops; there is no guarantee that scientific experiments can be repeated exactly because even when we sit, we travel in universe every second, hence, if we miss something, we can't have it back; however, this does not prevent us from making use of upcoming quantum product [6].

\section{References}

[1] R. Perlner, D. Smith-Tone, A Classification of Differential Invariants for Multivariate Postquantum Cryptosystems, PQCrypto 2013.

[2] National University of Singapore, Centre for Quantum Technologies Annual Report, 2008.

[3] ChunNina Ren, JianQi Zhang, Libo Chen and YongJian Gu, Optomechanical steady-state entanglement induced by electrical interaction, arXiv, 2014.

[4] Matthäus Halder, Alexios Beveratos, Nicolas Gisin, Valerio Scarani, Christoph Simon \& Hugo Zbinden, Entangling Independent Photons by Time Measurement, Swiss NCCR Quantum Photonics Project Report, 2008.

[5] Imam Usmani, Christoph Clausen, Felix Bussieres, Nicolas Sangouard, Mikael Afzelius and Nicolas Gisin, Heralded quantum entanglement between two crystals, arXiv 2011.

[6] Barry Olney, Entanglement: the holy grail of high-speed design, The PCB Design Magazine, 2014. 